\title{
Editorial
}

\section{Reviewer Appreciation Editorial}

\section{Seung-Woo Jeong ${ }^{\dagger}$}

Editor-In-Chief, Journal of Korean Society of Environmental Engineers, Republic of Korea

(Received January 21, 2022; Accepted January 21, 2022)

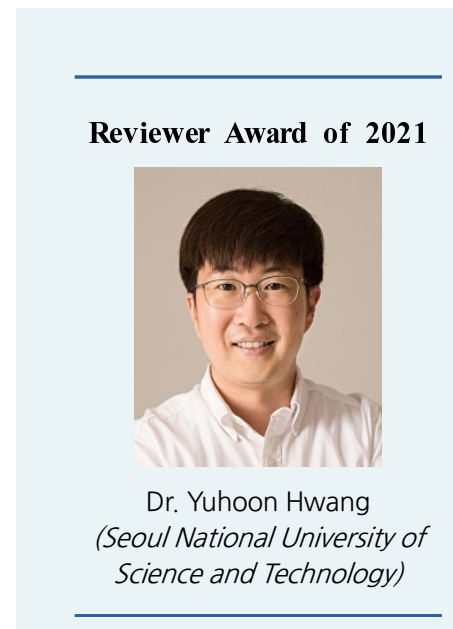

In 2021, a total of 67 papers were published in JKSEE. A total of 106 dedicated reviewers carefully reviewed the papers of this journal. Their constructive reviews assisted both the authors to improve the works and the editors to produce timely high-quality articles. Many reviewers answered the review request multiple times. Once a year I would like to thank these anonymous reviewers for their willingness and readiness.

The Korea Society of Environmental Engineers (KSEE) has established "Reviewer Award" since 2018 and awards the best reviewer every year. The Reviewer Award of 2021 is given to Dr. Yoohun Hwang (Seoul National University of Science and Technology). I sincerely appreciate the reviewers listed below for their time and contribution.

\section{Exemplary Reviewers 2021}

Ahn, Chang Hyuk

Korea Institute of Science and Technology

Ahn, Changmin

Seoul Waterworks Authority

Ahn, Hae Young

Sejong University

Ahn, Jae Chan

Seoul Waterworks Authority

Ahn, Jae hwan

Korea Institute of Science and Technology

Ahn, Yongtae

Gyeongnam National University of Science and

Technology

An, Byungryul

Sangmyung University

An, Jinsung

Semyung University

Bae, Chang Han

National Air Emission Inventory and Research

Center
Bae, Eun Young

Busan Water Authority

Bae, Sungjun

Konkuk University

Cha, Yoonkyung

University of Seoul

Chae, Kyujung

Korea Maritime \& Ocean University

Chang, Seo II

University of Seoul

Cho, II hyoung

Kyonggi University

Cho, Kyung Hwa

Ulsan National Institute of Science and Technology

Cho, Kyung-Suk

Ewha Womans University

Cho, Si-Kyung

Dongguk University

Choi, Hee-Jeong

Catholic Kwandong University
Choi, Hyeoksun

Wonkwang University

Choi, June-Seok

Korea Institute of Science and Technology

Choi, Suk Soon

Semyeong University

Choi, Sung-Deuk

Ulsan National Institute of Science and Technology

Choi, Young Gyun

Chungnam National University

Choi, Youngik

Dong-A University

Chon, Kangmin

Kangwon National University

Chung, Se-Woong

Chungbuk National University

Gopalakrishnan Kumar

University of Stavanger

Han, Changseok

Inha University 


\begin{tabular}{|c|c|c|}
\hline $\begin{array}{l}\text { Hur, Jin } \\
\text { Sejong University }\end{array}$ & $\begin{array}{l}\text { Kim, Junbeum } \\
\text { University of Technology of Troyes, France }\end{array}$ & $\begin{array}{l}\text { Lim, Seong-Rin } \\
\text { Kangwon National University }\end{array}$ \\
\hline $\begin{array}{l}\text { Hwang, Yuhoon } \\
\text { Seoul National University of Science and Technology }\end{array}$ & $\begin{array}{l}\text { Kim, Kitae } \\
\text { Seoul National University of Science and Technology }\end{array}$ & $\begin{array}{l}\text { Moon, Hee Sun } \\
\text { Korea Institute of Geoscience and Mineral Resources }\end{array}$ \\
\hline $\begin{array}{l}\text { Hyun, Seunghun } \\
\text { Korea University }\end{array}$ & $\begin{array}{l}\text { Kim, Kwang Soo } \\
\text { Korea Institute of Science and Technology }\end{array}$ & $\begin{array}{l}\text { Myung, Jaewook } \\
\text { Korea Advanced Institute of Science and Technology }\end{array}$ \\
\hline $\begin{array}{l}\text { Jang, Jae Kyung } \\
\text { National Institute of Agricultural Sciences }\end{array}$ & $\begin{array}{l}\text { Kim, Sang-Hyoun } \\
\text { Yonsei University }\end{array}$ & $\begin{array}{l}\text { Oh, Hyun-Suk } \\
\text { Seoul National University of Science and Technology }\end{array}$ \\
\hline $\begin{array}{l}\text { Jeon, Joonho } \\
\text { Changwon National University }\end{array}$ & $\begin{array}{l}\text { Kim, Suhan } \\
\text { Pukyong National University }\end{array}$ & $\begin{array}{l}\text { Pak, Daewon } \\
\text { Seoul National University of Science and Technology }\end{array}$ \\
\hline $\begin{array}{l}\text { Jeon, Jun Min } \\
\text { Green Environmental Complex Center }\end{array}$ & $\begin{array}{l}\text { Kim, Yongjin } \\
\text { Mokpo National Maritime University }\end{array}$ & $\begin{array}{l}\text { Par, Seong Jik } \\
\text { Hankyong National University }\end{array}$ \\
\hline $\begin{array}{l}\text { Jeon, Tae wan } \\
\text { National Institute Of Environmental Research }\end{array}$ & $\begin{array}{l}\text { Kim, Young II } \\
\text { Chaungnam Institute }\end{array}$ & $\begin{array}{l}\text { Park Donghee } \\
\text { Yonsei University }\end{array}$ \\
\hline $\begin{array}{l}\text { Jeong, Sanghyun } \\
\text { Pusan National University }\end{array}$ & $\begin{array}{l}\text { Kim, Young Mo } \\
\text { Hanyang University }\end{array}$ & $\begin{array}{l}\text { Park, Jae Woo } \\
\text { Hanyang University }\end{array}$ \\
\hline $\begin{array}{l}\text { Jeong, Seung-Woo } \\
\text { Kunsan National University }\end{array}$ & $\begin{array}{l}\text { Koo, Bonyoung } \\
\text { Chonnam National University }\end{array}$ & $\begin{array}{l}\text { Park, Jeryang } \\
\text { Hongik University }\end{array}$ \\
\hline $\begin{array}{l}\text { Jho Eun Hea } \\
\text { Chonnam National University }\end{array}$ & $\begin{array}{l}\text { Koo, Ja-Ho } \\
\text { Yonsei University }\end{array}$ & $\begin{array}{l}\text { Park, Jungyu } \\
\text { Chosun University }\end{array}$ \\
\hline $\begin{array}{l}\text { Joe, Woo-Hyeun } \\
\text { Seoul Waterworks Authority }\end{array}$ & $\begin{array}{l}\text { Kuppam Chandrasekhar } \\
\text { Yonsei University }\end{array}$ & $\begin{array}{l}\text { Park, Kyung-Soo } \\
\text { Institute for Advanced Engineering }\end{array}$ \\
\hline $\begin{array}{l}\text { Joo, Jin Chul } \\
\text { Hanbat National University }\end{array}$ & $\begin{array}{l}\text { Kwak, Jin II } \\
\text { Konkuk University }\end{array}$ & $\begin{array}{l}\text { Park, Seong Kyu } \\
\text { Kfene CO., LTD. }\end{array}$ \\
\hline $\begin{array}{l}\text { Jung, Kwang-Wook } \\
\text { Korea Water Environment Research Institute }\end{array}$ & $\begin{array}{l}\text { Kwon, Eilhann } \\
\text { Sejong University }\end{array}$ & $\begin{array}{l}\text { Park, Sung Hyuk } \\
\text { Dong-A University }\end{array}$ \\
\hline $\begin{array}{l}\text { Jung, Sokhee } \\
\text { Chonnam National University }\end{array}$ & $\begin{array}{l}\text { Lee, Byung Joon } \\
\text { Kyungpook National University }\end{array}$ & $\begin{array}{l}\text { Prakash Gautam } \\
\text { Kunsan National University }\end{array}$ \\
\hline $\begin{array}{l}\text { Kang, Hojeong } \\
\text { Yonsei University }\end{array}$ & $\begin{array}{l}\text { Lee, Changha } \\
\text { Seoul National University }\end{array}$ & $\begin{array}{l}\text { Rishikesh Bajagain } \\
\text { Kunsan National University }\end{array}$ \\
\hline $\begin{array}{l}\text { Kang, Seoktae } \\
\text { Korea Advanced Institute of Science and Technology }\end{array}$ & $\begin{array}{l}\text { Lee, Dae Sung } \\
\text { Kyungpook National University }\end{array}$ & $\begin{array}{l}\text { Seo, Jee-Won } \\
\text { Korea Water and Wastewater Works Association }\end{array}$ \\
\hline $\begin{array}{l}\text { Kim, Chang Gyun } \\
\text { Inha University } \\
\text { Kim, Chulhwan }\end{array}$ & $\begin{array}{l}\text { Lee, Dongsub } \\
\text { Kyungwoon University } \\
\text { Lee, Eui-Jong }\end{array}$ & $\begin{array}{l}\text { Shin, Seung Gu } \\
\text { Gyeongnam National University of Science and } \\
\text { Technology }\end{array}$ \\
\hline Korea Expressway Corporation & Daegu University & Son, Sunghoon \\
\hline $\begin{array}{l}\text { Kim, Dong-Jin } \\
\text { Hallym University }\end{array}$ & $\begin{array}{l}\text { Lee, Heekwan } \\
\text { Incheon National University }\end{array}$ & $\begin{array}{l}\text { Chonnam National University } \\
\text { Tirath Raj }\end{array}$ \\
\hline $\begin{array}{l}\text { Kim, I Tae } \\
\text { Korea Institute of Science and Technology }\end{array}$ & $\begin{array}{l}\text { Lee, jaejun } \\
\text { Jeonbuk National University }\end{array}$ & $\begin{array}{l}\text { Yonsei University } \\
\text { Yang, Jae Kyu }\end{array}$ \\
\hline $\begin{array}{l}\text { Kim, ljung } \\
\text { Hongik University }\end{array}$ & $\begin{array}{l}\text { Lee, Kyoungbin } \\
\text { Ministry of Environment }\end{array}$ & $\begin{array}{l}\text { Kwangwoon University } \\
\text { Yeon, In Sung }\end{array}$ \\
\hline $\begin{array}{l}\text { Kim, Jin Keun } \\
\text { Jeju National University }\end{array}$ & $\begin{array}{l}\text { Lee, Sungyun } \\
\text { Kyungpook National University }\end{array}$ & $\begin{array}{l}\text { Sangmyung University } \\
\text { Yoo, Chang Kyoo }\end{array}$ \\
\hline $\begin{array}{l}\text { Kim, Jin Sik } \\
\text { National Air Emission Inventory and Research Center }\end{array}$ & $\begin{array}{l}\text { Lee, Tae ho } \\
\text { Pusan National University }\end{array}$ & $\begin{array}{l}\text { Kyung Hee University } \\
\text { Yoo, Kyuseon }\end{array}$ \\
\hline $\begin{array}{l}\text { Kim, Jong Bum } \\
\text { Chungnam Institute }\end{array}$ & $\begin{array}{l}\text { Lee, Wontae } \\
\text { Kumoh National Institute of Technology }\end{array}$ & $\begin{array}{l}\text { Jeonju University } \\
\text { Yoon, Junheon }\end{array}$ \\
\hline $\begin{array}{l}\text { Kim, Jong Kyu } \\
\text { Shinhan University }\end{array}$ & $\begin{array}{l}\text { Lee, Yong Woon } \\
\text { Chonnam National University }\end{array}$ & $\begin{array}{l}\text { National Institute of Chemical Safety } \\
\text { Yun, Yeo-Myeong }\end{array}$ \\
\hline $\begin{array}{l}\text { Kim, Joung Dae } \\
\text { Hallym Polytechnic University }\end{array}$ & $\begin{array}{l}\text { Lim Dong-Hee } \\
\text { Chungbuk National University }\end{array}$ & Chungbuk National University \\
\hline
\end{tabular}

\section{Authors}

\section{Seung-Woo Jeong}

Journal of Korean Society of Environmental Engineers, Editor-In-Chief, Department of Environmental Engineering, Kunsan National University, Professor, ORCiD [0] 0000-0001-7418-4576 